\title{
Reflexiones sobre los desafíos que enfrentan las académicas en el contexto del capitalismo académico
}

\author{
Carmen A. Araneda-Guirriman*1 y Geraldy L. Sepúlveda-Páez ${ }^{2}$ \\ (1) Facultad de Ingeniería, Depto. de Ingeniería Industrial y de Sistemas, Universidad de Tarapacá, Arica, Chile \\ (correo-e: caraneda@academicos.uta.cl) \\ (2) Universidad de Tarapacá, 18 de septiembre \#2222, Arica, Chile. (correo-e: geraaldy@gmail.com) \\ * Autor a quien se debe dirigir la correspondencia.
}

Recibido Feb. 11, 2021; Aceptado Abr. 7, 2021; Versión final May. 13, 2021, Publicado Oct. 2021

\begin{abstract}
Resumen
El objetivo de este estudio es realizar un análisis conceptual sobre los desafíos que enfrentan las mujeres a lo largo de su trayectoria académica, en un contexto donde predomina la racionalidad, el desempeño, la mercantilización y el capitalismo académico. Para este fin, se presenta una revisión de artículos teóricos y empíricos de corriente principal de los últimos 5 años. Como resultado, se identificaron 4 principales desafíos: 1) la desigualdad sostenida que enfrenta la mujer en la educación superior, 2) el efecto negativo del capitalismo académico sobre las trayectorias académicas de las mujeres, 3) la posición hegemónica de la masculinidad en la academia, y 4) la conciliación entre la vida familiar y laboral que experimentan las mujeres. Se concluye que las acciones propias del capitalismo académico son procesos de género con efectos sobre las trayectorias académicas del profesorado universitario femenino.
\end{abstract}

Palabras clave: mujeres académicas; trayectorias; desafíos; capitalismo académico

\section{Reflections on the challenges faced by female academics in the context of academic capitalism}

\begin{abstract}
The primary aim of the present study is to perform a conceptual analysis of the challenges faced by women throughout their academic careers in a context where rationality, performance, commercialization, and academic capitalism are dominant. To this end, a review of mainstream theoretical and empirical articles from the last five years is presented. As a result, four main challenges are identified: 1) sustained inequality faced by women in higher education, 2) negative effect of academic capitalism on women's academic careers, 3) predominant positioning of masculinity in academia, and 4) reconciliation between work and family life experience by women. It is concluded that academic capitalism generates gender-based processes that affect the academic trajectories of female academics.
\end{abstract}

Keywords: women faculty; academic trajectories; challenges; academic capitalism 


\section{INTRODUCCIÓN}

La educación superior ha experimentado cambios que han reestructurado las instituciones, los sistemas de educación superior con variaciones en la política de investigación y desarrollo que han movilizado a las universidades hacia el capitalismo académico (Slaughter, 2018), donde el poder en las instituciones cambia de estar basado en el estatus social hacia uno sustentado en el capital comercializable (Schulze-Cleven y Olson, 2017). Esto implica un incremento en las demandas específicas de productividad, como publicaciones revisadas por pares y la adopción de comportamientos casi empresariales, con el fin generar ingresos a través de las patentes, la subvención de la investigación y los derechos de autor (Lester y Sallee, 2017).

El capitalismo académico sintetiza cómo las autoridades públicas dirigen la educación superior hacia los procesos de creación de valor, como en la economía privada, al fomentar acciones particulares de desempeño (Schulze-Cleven y Olson, 2017). Por lo tanto, el capitalismo académico en la educación superior se concreta a través de la institucionalización de la universidad operativa y se explicita por medio de las políticas de ciencia, tecnología e innovación en una acción conjunta entre actores internos (decanos, profesores y estudiantes) y externos, donde se encuentran representantes del gobierno, corporaciones, fundaciones privadas entre otros (Sigahi y Satorato, 2018).

En este contexto, O`Hagan et al (2019) establece que son los cuerpos masculinos los más capacitados en las prácticas profesionales que permiten lograr el capital académico, mientras que las mujeres se encuentran en desventaja y están menos preparadas para este tipo de prácticas. Se puede establecer la existencia de una subestructura de género, que devela los procesos organizacionales donde las creencias sobre hombres y mujeres, feminidad y masculinidad, se sostienen, son reproducidas y las desigualdades perpetuadas (Acker, 2012). Al respecto, Liang y Lin (2021) plantean que la universidad como organización no es neutra en cuanto al género, puesto que estas buscan un sujeto libre, racional y autónomo capaz de trabajar eficientemente sin otras responsabilidades, por tanto la división desigual del trabajo doméstico y sus responsabilidades generan una situación de desventaja para las mujeres que inician su carrera académica en el contexto de la educación superior contemporánea.

Asimismo, las prácticas académicas producen efectos de género, ante lo cual las profesoras universitarias deben estar atentas respecto a los impactos que tiene, una academia masculinizada y predominantemente anglosajona, en la producción de conocimiento y en las rutinas del trabajo académico esperado (Caretta et al., 2018), situación que está invisibilizada y naturalizada al interior de esta esfera. Particularmente, por las múltiples tensiones que experimentan las mujeres en lo referente a la conciliación de la familia y la carrera. Resulta importante, por lo tanto, monitorear la situación de las mujeres, sobre todo al inicio de su carrera académica, con el fin de facilitar las oportunidades de desarrollo a través redes de apoyo entre pares y mentores sénior (Meschitti, 2020).

Ferree y Zippel (2015) plantean que el capitalismo académico corre el riesgo de incorporar medidas de igualdad de género en una agenda de mercado, pero con una perspectiva de género que se encuentra débilmente posicionada como agente crítico. El capitalismo académico, por consiguiente, en el actual escenario puede ser utilizado como una teoría de género, pues logra explicar cómo el predominio masculino se está afianzando cada vez más en las instituciones de educación superior mediante una agenda económica racional, a pesar de los logros modestos o significativos de las mujeres (Metcalfe y Slaughter, 2011).

Reflexionar en torno a la posición y las condiciones que tienen las mujeres en la academia actual resulta necesario, puesto que los desafíos que deben enfrentar son diversos y complejos, más aún cuando predomina el capitalismo académico. El escenario actual de la educación superior conlleva una serie de exigencias adicionales, de competitividad y excelencia, las cuales repercuten en todo el quehacer académico. Por ende, estas exigencias también impactan en las trayectorias académicas femeninas, al considerar solo ciertos aspectos como relevantes, como sucede con la investigación de prestigio y la productividad académica, procesos en los cuales las mujeres tienen menor participación.

Por lo tanto, el objetivo de este trabajo es presentar un análisis conceptual, de los principales desafíos que enfrentan las profesoras universitarias en sus carreras académicas. Este análisis considera el actual contexto de la educación superior en que predomina el capitalismo académico. Para este fin se efectúa una revisión secundaria de artículos científicos teóricos y empíricos de corriente principal de los últimos 5 años.

\section{METODOLOGÍA}

La presente investigación corresponde a una revisión documental teórica de artículos de corriente principal respecto al género, capitalismo académico y trayectorias académicas del profesorado femenino, con la finalidad de comprender de mejor forma los desafíos que enfrentan las académicas en la actualidad. La revisión conceptual consideró artículos teóricos y empíricos que abordaban el contexto del capitalismo académico en la educación superior y las exigencias que trae consigo para el cuerpo académico. Esto con el 
fin de aportar en la identificación de aquellos elementos críticos de la academia que se transforman en desafíos para el profesorado universitario femenino. Para este fin, el énfasis en la revisión conceptual de los 30 artículos científicos más relevantes que abordaban dicha problemática en los últimos 5 años. De este modo, se logra identificar y describir el contexto en el que están inmersas las académicas, su proceso de incorporación a la academia, su representatividad en las áreas del conocimiento que valora el capitalismo académico y participación en áreas del conocimiento, la productividad académica y conciliación familiarlaboral.

\section{REVISIÓN CONCEPTUAL}

A continuación, se presenta la revisión conceptual que aborda el contexto de la educación superior caracterizado principalmente por la presencia del capitalismo académico, para posteriormente revisar conceptualmente los desafíos que enfrentan las académicas en el escenario previamente señalado.

\section{Contexto de la educación superior}

El capitalismo académico refleja los comportamientos, cambios y procesos que guían a la educación superior hacia un escenario donde predomina la lógica del mercado en los niveles macroestructurales, organizacionales e individuales (Sigahi y Saltorato, 2020). La teoría del capitalismo académico observa, por lo tanto, como un grupo de actores (estudiantes, académicos, administradores) usa una variedad de recursos estatales, con el fin de crear nuevos circuitos de conocimiento que vinculan a las instituciones de educación superior con la nueva economía (Slaughter y Rhoades, 2004:14). Por consiguiente, involucra nuevos circuitos en la producción y difusión del conocimiento que se sustentan en una lógica distintiva de mercado (Rhoades y Torres-Olave, 2015).

El capitalismo académico permite, por ende, definir la forma en que las universidades públicas, orientadas a la investigación, responden a las tendencias que consideran a la educación superior como un subconjunto dentro de la política económica (Slaughter y Leslie, 2001). La ética del mercado predominante convirtió a la educación superior en un tema de negocios, y a la ciencia en una fuerza productiva, con énfasis en la universidad emprendedora y el culto al desempeño que intensifican la propia expansión del capitalismo (Sigahi y Saltorato, 2020). Del mismo modo, el capitalismo académico tiene sus contradicciones, pues en las universidades públicas dedicadas a la investigación los académicos son empleados de la universidad y a su vez empleados autónomos y competitivos que actúan como capitalistas dentro del sector público (Slaughter y Leslie, 2001).

El enfoque del capitalismo académico permite, por lo tanto, comprender la situación de las mujeres en la academia, en un escenario predominado por el género masculino en áreas como la ciencia e ingeniería que cuentan con los mayores ingresos; mientras que las mujeres están en mayor número en las áreas del bienestar y las ciencias sociales (Metcalfe y Slaugther, 2011). Las áreas de ciencia, ingeniería, tecnología y matemática (STEM) a su vez son las adecuadas para el mercado, debido al potencial de comercialización de la ciencia, la investigación y su impacto en el crecimiento económico (O Hagan et al., 2019). La baja representación de las mujeres en el área de STEM puede explicarse por la asignación de las académicas a las actividades de servicios y comités, mentorías, lo cual les resta tiempo para la investigación y por tanto afectan negativamente en el número de publicaciones, la obtención de recursos para investigar y el avance de su carrera académica (Casad et al., 2021). Estas labores asociadas al servicio y al cuidado del lugar de trabajo se engloban en lo que la literatura denomina trabajo doméstico académico (Heijstra et al. 2017).

\section{Incorporación de las mujeres a la academia}

El sistema de educación superior se basa en los principios de la igualdad, el mérito y la capacidad, no obstante, las mujeres a finales del siglo XIX luchaban por ser aceptadas en las universidades. En la actualidad, a pesar del incremento que ha tenido la participación de las mujeres en la educación superior, este no ha significado una situación de igualdad de oportunidades con respecto a los hombres, puesto que la desigualdad de género en esta esfera aún persiste (Montes-López y Groves, 2019), lo cual se configura como uno de los principales desafíos para las mujeres.

En este sentido, la educación superior tradicionalmente ha estado vinculada a los hombres de clase media y alta. Hasta la década de los años 70 las mujeres estuvieron excluidas o sujetas a cuotas de participación, con poca presencia en la academia y en las universidades de investigación. Posteriormente, cuando los movimientos sociales de mujeres consiguieron un espacio en la academia, los hombres se vieron obligados a compartir sus privilegios, sin embargo, no fue una situación de ganar/ganar, puesto que las mujeres pretendían expandir su número en los puestos profesionales, académicos y de gestión, lo cual finalmente no sucedió (Metcalfe y Slaugther, 2011). 
Ejemplo de esto se puede considerar el caso de la Universidad de California, Berkeley, cuyos departamentos dominados por mujeres (educación, biotecnología, enfermería, bienestar social y economía doméstica) comenzaron a principios del siglo XX como instituciones separadas, desiguales y de bajo prestigio, como un destino para ubicar el creciente número de mujeres y profesoras. Estos departamentos contaban con poco apoyo y fueron cerrados 50 años después, cuando ya no eran útiles, y aquellos que lograron permanecer lo hicieron sin recursos, ni prestigio y ante una crisis eran los primeros en ser cerrados (Nerad, 1999). La participación de las mujeres en la educación superior, por ende, no ha implicado una integración a la vida institucional en igualdad de circunstancias (Montes-López y Groves, 2019). A pesar de que el personal académico y profesional de las universidades está cada vez más feminizado, continúan persistiendo las segmentaciones por género en ambas profesiones, puesto que las mujeres se ubican mayoritariamente en los rangos académicos más bajos (Blackmore, 2020). Estas diferencias y desigualdades, entre hombres y mujeres que forman parte del cuerpo académico, se configuran como uno de los principales desafíos que persisten en el ámbito de la educación superior.

Es más, con el avance de las mujeres en la educación superior, las diferencias se han acentuado, puesto que los hombres se volvieron más activos en la construcción del régimen académico capitalista del conocimiento, lo cual resulta como un esfuerzo estratégico para continuar con sus históricos privilegios (Metcalfe y Slaughter, 2011). Estas diferencias de género entre hombres y mujeres que forman parte del cuerpo académico se manifiestan en el capital social, el impacto de las relaciones personales, la división del trabajo doméstico y las necesidades de los hijos las que repercuten principalmente en las mujeres al momento de solicitar un ascenso (Lipton, 2020).

\section{Representatividad y participación}

Con relación a la representatividad y participación de las mujeres en el mundo académico se puede establecer que el capitalismo académico está re-masculinizando la academia al definir un orden de género con prácticas efectuadas por hombres que mantienen esta estructura, junto a una baja participación de las mujeres en las facultades de ciencia, tecnología, ingeniería y matemática (O`Hagan et al., 2019). Áreas que por cierto concentran principalmente los fondos destinados para la investigación, tanto en Estados Unidos como en la Unión Europea (Slaughter y Cantwell, 2012). Por el contrario, las estudiantes y las académicas se concentran en las áreas de las humanidades, educación, ciencias sociales, artes y salud, donde experimentan sentimientos de inseguridad ante la priorización que se otorga a STEM en las universidades (Blackmore, 2020).

Este énfasis otorgado a las áreas de ciencia e ingeniería, debido a su capacidad para generar recursos y estatus, puede provocar diferencias de género en los ingresos que recibe el cuerpo académico (Johnson y Taylor, 2019). Estas diferencias son las que generan prácticas profesionales de género y que conforman el mecanismo, por medio del cual se perpetúa el capitalismo académico y se refuerzan las diferencias de género (O`Hagan, 2019). Leathwood (2017), por su parte señala que las mujeres han sido históricamente vistas como intrusas en la educación superior, puesto que las académicas continúan marginalizadas en la economía de la investigación y excluidas de las posiciones de investigación sénior, reciben menos fondos y tienen menos probabilidades de que su investigación sea considerada de excelencia, situación que podría facilitar la existencia de diferencias de género en la compensación del cuerpo académico (Johnson y Taylor, 2019).

De igual modo, el discurso de la masculinidad, en la cultura organizacional de la universidad, se manifiesta por el capitalismo académico que facilita las condiciones para que los académicos recapturen parte de su histórico privilegio derivado de la educación superior (Metcalfe y Slaughter, 2011). Es así como Kandiko, Coate y de St Croix (2017) sostienen que el prestigio se considera como un concepto de género, puesto que sus logros y recompensas tienden a favorecer a los hombres y desfavorecer a las mujeres, quienes están escasamente representadas en los cuerpos editoriales, sus publicaciones son menos citadas y en menor medida son invitadas como oradores principales en seminarios y congresos. Por lo tanto, se requieren esfuerzos para incrementar la participación de las mujeres en la presentación de manuscritos, con el fin de permitir su avance y retención en la ciencia (Lerback y Hanson, 2017). Esto resulta fundamental para que las académicas puedan desenvolverse en un ambiente en el que impera la lógica del capitalismo académico, donde las universidades no solo compiten por financiamiento, sino que también por prestigio (Johnson y Taylor, 2019). Por lo tanto, la metricización y la mercantilización de la universidad son procesos con diferencias en género (Thornham, 2017).

\section{Productividad académica}

El capitalismo académico ha tenido impactos en las exigencias y desafíos que debe enfrentar el cuerpo académico, puesto que demanda determinadas formas de productividad, tales como: publicaciones con revisión de pares, fondos de investigación, patentes, entre otros. A su vez, requiere la separación de los roles 
correspondientes, por una parte, a las funciones docentes, y a las actividades de investigación, por otra (Lester y Sallee, 2017).

En este contexto, principalmente se encuentran las mujeres en la docencia, mientras que los hombres están en la creación de capital académico por medio del emprendimiento tecnológico, lo cual evidencia una remasculinización de la academia y del ideal académico (Thornton, 2013). Asimismo, las mujeres son quienes tienen menos vínculos con la industria y, por ende, tienen menos probabilidades de recibir financiación de su parte, en comparación a sus colegas masculinos, por lo tanto, se requiere especial atención sobre esto (Sigahi y Saltorato, 2020). Particularmente, cuando los nuevos circuitos del conocimiento en el capitalismo académico se caracterizan por operar con una lógica de mercado consistente en un aumento de las publicaciones, patentes, licencias y actividades emprendedoras (Slaughter y Cantwell, 2012). Por consiguiente, resulta crucial la participación de las mujeres en las actividades académicas relevantes para el desarrollo y la retención laboral, como lo son la revisión de publicaciones, la participación en conferencias, la obtención de subvenciones, las nominaciones a premios, las colaboraciones profesionales y el ser parte de las decisiones de contratación o subvención (Lerback y Hanson, 2017).

Para la academia es, por lo tanto, un desafío el incentivar la participación de las mujeres en dichas actividades que la involucren en la investigación, puesto que como señalan Liang y Lin (2021) en la actualidad para lograr la titularidad en la academia universitaria resulta mucho más importante la investigación que la docencia. Más aún si se considera que es más común el aumento en la creación de puestos de trabajos para el profesorado femenino se encuentra orientado particularmente a la docencia (Thornton, 2013). En este sentido, los resultados de una investigación efectuada por Véliz (2019) muestran que para las académicas extranjeras los asuntos de género forman parte de sus experiencias, y en muchos casos, el ser mujer no fue percibido como una ventaja en sus carreras. Por el contrario, los resultados de un estudio realizado por O`Hagan et al (2019) indican que los hombres al ser consultados sobre el impacto de su género en sus carreras académicas respondieron que no había tal impacto y se manifestaron desconcertados con la pregunta, pues nunca lo habían considerado, situación que sugiere que los hombres desconocen su privilegio masculino.

Por tanto, el capitalismo académico ha facilitado las condiciones para que los hombres recuperasen en las universidades algunos de los privilegios derivados de la educación superior y a la vez reformulasen su valor en este proceso (Metcalf y Slaughter, 2011). En consecuencia, el aumento de la representación del género femenino oculta el hecho de que la participación de las mujeres continúa siendo medida y evaluada con relación a las normas y parámetros masculinos, asimismo, las mujeres permanecen en gran parte invisibles como líderes académicas y respetadas productoras de conocimiento (Lipton, 2020).

\section{Conciliación vida familiar y laboral}

Los resultados obtenidos por Thun (2018) evidencian que la academia en las universidades noruegas presenta puntos ciegos y sesgos vinculados al género y al estado parental. Estos sesgos afectan principalmente a las mujeres, debido a que los cambios introducidos por el capitalismo académico rompen el equilibrio entre el trabajo y la vida personal. Por lo tanto, para comprender la persistencia de las desigualdades de género en la academia resulta clave reconocer las condiciones organizativas para la maternidad. En consecuencia, como señala Harris et al. (2019) analizar las carreras académicas de quienes son padres/madres, resulta crucial, puesto que se configura como uno de los desafíos identificados por el profesorado femenino que posee una trayectoria académica exitosa, al ser principalmente las mujeres quienes tienen las responsabilidades del cuidado doméstico (Liang y Lin, 2021).

De acuerdo con Morley (2018), en la educación superior las mujeres han discutido respecto al mérito, el cual funciona como un refuerzo para la ventaja masculina, pues recompensa aspectos que ellos hacen bien, como sucede con la dedicación exclusiva a la investigación y publicación por periodos prolongados, con importantes oportunidades de patrocinio y desarrollo, y sin otras responsabilidades como el cuidado del hogar. Asimismo, O'Connor et al. (2017) en su estudio observó que tanto hombres como mujeres destacaron la imagen monástica del científico y su tensión con la vida familiar, puesto que las responsabilidades domésticas y afectivas impactan más a las mujeres. En efecto, las mujeres del cuerpo académico enfrentan el desafío de conciliar el trabajo y la familia (Véliz, 2019).

Leathwood (2017), a su vez señala que las mujeres reconocen que, para subsistir en la academia, deben dedicar largas horas de trabajo a la investigación, lo cual incorpora los fines de semana y el trabajo en horas de la noche. Desde esta perspectiva entonces se construye el ideal académico que considera la expectativa de estar totalmente dedicado al trabajo (Lester y Sallees, 2017). Por lo tanto, mantener la idea de que el género no juega ningún rol en la progresión académica de las mujeres, o en la capacidad de triunfar en el mercado de la educación superior, invisibiliza las desigualdades materiales y afectivas que experimentan las académicas en la universidad (Lipton, 2020). 
En efecto, desde su origen las universidades han estado vinculadas a la masculinización del conocimiento, donde los requerimientos de independencia y el rigor de las disciplinas son asociados a la imagen de un sujeto ideal, caracterizado como blanco, occidental y masculino (Thornham, 2017). Por consiguiente, la posición de un trabajo de género neutro, en la cual se asume que el trabajador es abstracto, incorpóreo en palabras de Acker (2012), que no tiene un cuerpo ni obligaciones afuera del lugar de trabajo, y por tanto está libre de cargas, y que sea capaz de trabajar de manera eficiente y sin otras responsabilidades y necesidades fuera del ámbito laboral (Liang y Lin, 2021). Este perfil, según Acker (2012), viene asociado a ciertas expectativas de comportamiento del trabajador en la que encajan de mejor forma los hombres, quienes son percibidos como trabajadores reales, debido a que tradicionalmente la mujer ha realizado el trabajo no remunerado liberando al hombre de dicho compromiso. En efecto, en el actual contexto la conciliación entre la vida familiar y laboral se configura como un desafío para las mujeres que forman parte del cuerpo académico.

\section{RESULTADOS Y DISCUSIÓN}

El actual contexto de la educación superior ha implicado una serie de exigencias que, tanto hombres como mujeres, deben enfrentar al ser parte del cuerpo académico. Sin embargo, estas exigencias no afectan a todos por igual, puesto que, a partir de la revisión documental efectuada, se puede establecer que las académicas se enfrentan a un conjunto de desafíos que no son experimentados de igual forma por sus homólogos masculinos.

En este ámbito, se identificaron 4 principales desafíos que enfrentan las mujeres que forman parte del mundo académico. El primer desafío corresponde la reciente incorporación de la mujer en la educación superior y su consecuente situación de desigualdad que persiste en la actualidad. Esta situación de desigualdad se ve acentuada por el contexto del capitalismo académico que define prácticas que son más fáciles de alcanzar por los académicos de género masculino. Asimismo, un segundo desafío se relaciona a la baja representatividad de las mujeres en las áreas del conocimiento de la ciencia e ingeniería, las cuales son predominantes en el contexto del capitalismo académico. En efecto, en las universidades de investigación las áreas de ciencia, tecnología, ingeniería y matemática continúan creciendo (Slaughter y Cantwell, 2012). Estas áreas históricamente han sido dominadas por hombres, con la consecuente definición de códigos y prácticas para su funcionamiento. Por lo tanto, las diferencias de género persisten y son reproducidas, pero están invisibilizadas.

De este modo, las exigencias de productividad académica se configuran como un tercer desafío, las cuales hacen referencia principalmente a las publicaciones académicas, la investigación, las patentes y el emprendimiento tecnológico. Actividades en las que participan en su mayoría los miembros masculinos del cuerpo académico, mientras que las mujeres suelen estar más orientadas a las labores de docencia y de servicio. Por lo tanto, es un desafío institucional incentivar la participación de las mujeres en las actividades de investigación y en aquellas instancias claves para el desarrollo académico, sin limitarlas únicamente a las labores de docencia y de servicio, es decir, al trabajo doméstico académico.

En consecuencia, se debe tener en cuenta que la representatividad y participación de las mujeres, en el mundo académico, ocurre en una estructura donde predominan los académicos y una posición hegemónica de la masculinidad. Por lo tanto, el capitalismo académico, en lugar de solo potenciar los ingresos externos, podría mejorar los beneficios de la propiedad y los servicios educativos, con mayores compromisos con las poblaciones en desventaja y el incremento de las oportunidades para las mujeres y las minorías (Slaughter y Rhoades, 2004).

Por último, se encuentra el cuarto desafío correspondiente a la conciliación entre la vida familiar y laboral que experimentan las mujeres, en particular sobre la maternidad y las responsabilidades de cuidado del hogar que, usualmente, recaen sobre el género femenino. La complejidad de esto se ve intensificada al considerar que las mujeres soportan de manera desproporcionada el trabajo no remunerado del cuidado del hogar, lo cual se ha hecho más evidente con la pandemia Covid-19 (Enguita-Fernández et al., 2020) junto con las exigencias de desempeño que trae consigo la actual academia. Esta situación a veces entra en conflicto con la imagen del "académico" que predomina en las universidades y que hace referencia a un sujeto dedicado por completo a la academia sin responsabilidades y preocupaciones fuera de este ámbito.

En efecto, durante el último año producto de la pandemia del Covid-19 se ha develado la dificultad de conciliar ambos aspectos, al exponer el rol que desempeñan en su mayoría las mujeres con relación al cuidado del hogar. Esto evidencia que en el espacio doméstico la división del trabajo por género se ha tardado en cambiar, lo cual causa conflictos en la negociación de los límites entre el trabajo y la familia, donde además el teletrabajo ha impuesto cargas desproporcionadas a las mujeres (Bahn et al., 2020). Por consiguiente, es posible establecer que en la modalidad de teletrabajo las mujeres tengan menos probabilidades de mantener altos niveles de productividad (Sigahi et al., 2021) los cuales se exigen al cuerpo académico en la actualidad. 
Sin embargo, aún faltan estudios que analicen en profundidad las implicancias de la pandemia Covid-19 en las trayectorias de las académicas femeninas, en particular en lo referente a las medidas de confinamiento, puesto que como señala Castañeda-Rentería (2021) el "estar en casa" retrasa los tiempos dedicados al trabajo, el cuidado y el autocuidado, lo que implica costos productivos y emocionales en las mujeres, quienes culturalmente han destinado su tiempo a otros que son receptores del cuidado directo o indirecto a través del cuidado del hogar. Esto demuestra, en palabras de la autora, la sub-representación de las tensiones que experimenta la mujer en cuanto a la distribución de su tiempo para el cumplimiento de diversas tareas. Al respecto, Blackmore, (2020) señala que, en la educación superior australiana, la atención que han otorgado las universidades en las áreas de educación y salud, ambas compuestas mayoritariamente por mujeres, se ha sustentado sobre la fuerza laboral femenina que trabaja a través de la interfaz mientras controla la educación de sus hijos en el hogar, cuya carga se intensificó con los encierros a los que se sometió la población para disminuir la propagación del virus.

El presente artículo muestra una revisión teórica respecto a los desafíos que enfrenta el profesorado femenino en el contexto del capitalismo académico en la educación superior. A este respecto, en primera instancia, el análisis de artículos de corriente principal estuvo delimitado por el número de referencias permitidas para este tipo de publicaciones, por lo tanto, no se incorporaron todos los artículos referentes a esta temática, sino que se consideraron sólo los más actuales y relevantes en cuanto a su aporte a la reflexión teórica. En segundo lugar, muchos de los artículos revisados analizan la situación de las académicas en circunstancias normales, por lo tanto, solo una parte reducida de estos artículos publicados en 2020 y 2021 están incorporando los efectos de la pandémica Covid-19 en la trayectoria académica del profesorado femenino. Por último, cabe señalar que los antecedentes presentados corresponden a la etapa de actualización bibliográfica de una investigación en curso.

\section{CONCLUSIONES}

A partir de la revisión documental de fuentes secundarias teóricas y empíricas que abordan la situación de la mujer en la academia actual, se pueden señalar las siguientes conclusiones:

1. Las mujeres que forman parte de la academia en las universidades han debido enfrentar múltiples desafíos desde su incorporación en la esfera de la educación superior, lo cual es un hecho relativamente reciente que se desencadenó durante el siglo pasado.

2. La posición de las mujeres en el mundo académico es afectada por el contexto en donde se circunscribe el sistema de educación superior, puesto que logra determinar en buena parte el funcionamiento de las universidades dentro de este sistema. En la actualidad, este contexto se caracteriza por la presencia del capitalismo académico que establece un fuerte énfasis en las medidas de desempeño, eficiencia, y eficacia, con una orientación al mercado, lo cual impacta tanto en los niveles estructurales como individuales, debido a que trae consigo nuevas formas de producir el conocimiento.

3. Las exigencias asociadas a las formas de productividad científica mediante las publicaciones revisadas por pares investigación de prestigio que se esperan en el contexto del capitalismo académico son abordados con una perspectiva y estándares masculinos, ya que, las mujeres aún están sub-representadas en áreas claves como las ciencia, tecnología, ingeniería y matemática, donde se genera la mayor producción de conocimiento y el capital académico. Asimismo, las académicas se encuentran principalmente en la docencia y en las labores de servicio, por lo tanto, resulta clave su participación en las actividades críticas que le permitan participar en la investigación, la cual debe ser intencionada al interior de la institución.

4. La mantención de puntos ciegos con relación con el estado parental del cuerpo académico, limita la comprensión a cabalidad de la situación de las mujeres en la academia y más aún su participación en las formas de producir conocimiento y de conseguir capital académico. En efecto, dicha participación exige extensas jornadas y un arduo trabajo, lo cual muchas veces se dificulta, puesto que aún son ellas en su mayoría las responsables del cuidado del hogar y los hijos. Ciertamente, esta situación se ha intensificado durante la pandemia Covid-19, puesto que el confinamiento ha develado lo difícil que ha resultado para el profesorado femenino la conciliación. Sin embargo, el impacto de la pandemia en las trayectorias académicas del profesorado universitario femenino aún es un tema en desarrollo que debiera ser abordado en una futura investigación.

5. Todos estos elementos expuestos se configuran como desafíos, no solo para las mujeres que forman parte de la academia, sino que también para quienes definen políticas institucionales en pos de la consecución de capital académico altamente valorado en la actualidad, puesto que devela situaciones que posiblemente son invisibilizadas ante un discurso que asume dichos procesos como neutros en cuanto al género. En consecuencia, la participación del profesorado femenino en estas actividades debiera ser intencionada y propiciada al interior de la institución de educación superior. 


\section{AGRADECIMIENTOS}

Los autores reconocen y agradecen el patrocinio recibido por parte de ANID a través del proyecto FONDECYT $\mathrm{N}^{\circ} 1201517$, Mujeres en las altas jerarquías universitarias: percepciones de su trayectoria y experiencia académica a partir de sus propias voces.

\section{REFERENCIAS}

Acker, J., Gendered organizations and intersectionality: Problems and possibilities, https://doi.org/10.1108/02610151211209072, Equality, Diversity and Inclusion, 31 (3), 214-224 (2012)

Bahn, K., Cohen, J., y Rodgers, Y., A feminist perspective on COVID-19 and the value of care work globally, https://doi.org/10.1111/gwao.12459, Gender, Work \& Organization, 27(5), 695-699 (2020)

Blackmore, J., The carelessness of entrepreneurial universities in a world risk society: a feminist reflection on the impact of Covid-19 in Australia, https://doi.org/10.1080/07294360.2020.1825348, Higher Education Research \& Development, 39(7), 1332-1336 (2020)

Caretta, M. A., Drozdzewski, D., Jokinen, J. C., y Falconer, E., "Who can play this game?" The lived experiences of doctoral candidates and early career women in the neoliberal university, https://doi.org/10.1080/03098265.2018.1434762, Journal of Geography in Higher Education, 42(2), $261-275$ (2018)

Casad, B. J., Franks y otros 4 autores,. Gender inequality in academia: Problems and solutions for women faculty in STEM, https://doi.org/10.1002/jnr.24631, Journal of neuroscience research, 99(1), 13-23 (2021)

Castañeda-Renteria, L. I., The times of women and men. reflections on gender and time during the global health crisis; In Tempo e Sociedade em Suspenso / Time and Society in the Lounge by C, Sales., E, Araujo, y R, Costa. (Eds.) CIES, 21-34, Lisboa, Portugal (2021)

Enguita-Fernàndez, C., Marbán-Castro, E., y otros tres autores, The COVID-19 epidemic through a gender lens: what if a gender approach had been applied to inform public health measures to fight the COVID-19 pandemic?, https://doi.org/10.1111/1469-8676.12803, Social Anthropology, 28(2), 263-264 (2020)

Ferree, M. M., y Zippel K., Gender equality in the age of academic capitalism: Cassandra and Pollyanna interpret university restructuring, https://doi.org/10.1093/sp/jxv039, Social Politics: International Studies in Gender, State \& Society, 22(4), 561-584 (2015)

Harris, C., Myers, B., y Ravenswood, K., Academic careers and parenting: identity, performance and surveillance, https://doi.org/10.1080/03075079.2017.1396584, Studies in Higher Education, 44(4), 708-718 (2019)

Heijstra, T. M., Einarsdóttir, P., Pétursdóttir, G. M., y Steinpórsdóttir, F. S. Testing the concept of academic housework in a European setting: Part of academic career-making or gendered barrier to the top?.

https://doi.org/10.1177/1474904116668884, European Educational Research Journal, 16(2-3), $200-214$ (2017)

Johnson, J. A., y Taylor, B. J., Academic capitalism and the faculty salary gap, https://doi.org/10.1007/s10755-018-9445z, Innovative Higher Education, 44, 21-35 (2019)

Kandiko, C. B., Coate, K., y de St Croix, T., Mid-career academic women and the prestige economy, doi:10.1080/07294360.2017.1411337, Higher Education Research \& Development, 37(3), 533-548 (2017)

Leathwood, C., Women academic researchers: Still interlopers in the UK academy? In The changing role of women in higher education by Heather Eggins (Ed.) 17, 227-242. Springer, Cham. Switzerland (2017)

Lerback, J., y Hanson, B., Journals invite too few women to referee. Nature, 541(7638), 455-457 (2017)

Lester, J., y Sallee, M. W., Troubling gender norms and the ideal worker in academic life. In Critical Approaches to Women and Gender in Higher Education by P. L, Eddy., K, Ward., T, Khwaja (Eds.), Palgrave Macmillan, 115-138. New York, USA (2017)

Liang, L. F., y Lin, Y. H., The Struggle for "Survival" in Contemporary Higher Education: The Lived Experiences of Junior Academics in Taiwan. In The Palgrave Handbook of Institutional Ethnography by P.C, Luken, y S, Vaughan (eds.), Palgrave Macmillan, Cham, 259-279, Switzerland AG (2021)

Lipton, B., Academic women in neoliberal times. Palgrave Macmillan $1^{\text {st }}$ edition, 15-281 (2020)

Meschitti, V., Being an early career academic: is there space for gender equality in the neoliberal university?, https://doi.org/10.4337/9781786438973, In Gender, Science and Innovation. Edward Elgar Publishing, 11-34, Cheltenham, UK (2020)

Metcalfe, A., y S. Slaughter., Academic capitalism. In Gender and higher education. By B. Banks (Ed.) 13-20. The Johns Hopkins University Press, Baltimore, USA (2011)

Morley, L., Gender in the Neo-liberal Research Economy: An Enervating and Exclusionary Entanglement? In: Kahlert H. (eds) Gender Studies and the New Academic Governance, https://doi.org/10.1007/978-3-658-19853-4_2, 15-40, Springer VS, Wiesbaden (2018)

Montes-López, E., y Groves, T., Micro-machismo and discrimination in academia: The violation of the right to equality in university, https://doi.org/10.3989/chdj.2019.010, Culture \& Amp; History Digital Journal, 8(1), 1-9 (2019) 
Nerad, M., The academic kitchen: A social history of gender stratification at the University of California, Berkeley. SUNY Press. New York, United States of America (1999)

O'Connor, P., López, E. M., y otros diez autores. Micro-political practices in higher education: a challenge to excellence as a rationalising myth?, https://doi.org/10.1080/17508487.2017.1381629, Critical Studies in Education, 61(2), 1-17 (2017)

O'Hagan, C., O'Connor y otros siete autores. Perpetuating academic capitalism and maintaining gender orders through career practices in STEM in universities, https://doi.org/10.1080/17508487.2016.1238403, Critical Studies in Education, 60,2, 205-225 (2019)

Schulze-Cleven, T., y Olson, J. R., Worlds of higher education transformed: toward varieties of academic capitalism, https://doi.org/10.1007/s10734-017-0123-3, Higher Education, 73(6), 813-831 (2017)

Sigahi, T. F. A. C., y Saltorato, P., A emergência da Universidade Operacional: redes, liquidez e capitalismo académico, https://doi.org/10.1590/es0101-73302018187694, Educação \& Sociedade, 39(144), 522-546 (2018)

Sigahi, T. F. A. C., y Saltorato, P., Academic capitalism: distinguishing without disjoining through classification schemes, https://doi.org/10.1007/s10734-019-00467-4, Higher Education, 80 95-113 (2019)

Slaughter, S., Academic Capitalism, Evolution and Comparisons. In Encyclopedia of International Higher Education Systems and Institutions, by P, Teixeira., J, Shin (eds.), Springer, Netherlands 1-12 (2018).

Slaughter, S., Slaughter, S. A., \& Rhoades, G., Academic capitalism and the new economy: Markets, state, and higher education. JHU Press. Maryland, United States of America (2004)

Slaughter, S., y Cantwell, B., Transatlantic moves to the market: The United States and the European Union, https://doi.org/10.1007/s10734-011-9460-9, Higher Education, 63(5), 583-606 (2012)

Slaughter, S., y Leslie, L. L., Expanding and elaborating the concept of academic capitalism. https://doi.org/10.1177/1350508401082003, Organization, 8(2), 154-161 (2001)

Thornham, S., Reflections on equality, diversity, and gender at the end of a media studies headship, https://doi.org/10.1177/1527476417709342, Television \& New Media, 18(7), 689-696 (2017)

Thornton, M., The mirage of merit: Reconstituting the 'ideal academic', https://doi.org/10.1080/08164649.2013.789584, Australian Feminist Studies, 28 (76), 127-143 (2013)

Thun, C., Excellent and gender equal? Academic motherhood and 'gender blindness' in Norwegian academia, https://doi.org/10.1111/gwao.12368, Gender, Work \& Organization, 27(2), 166-180 (2019)

Véliz, D., Experiences of International Women Faculty at One Striving University, https://doi.org/10.1080/26379112.2019.1681006, Journal of Women and Gender in Higher Education, 13(1), 37-55 (2019) 
